Vertriebspower in turbulenten Zeiten 
Hartmut H. Biesel

\section{Vertriebspower in \\ turbulenten Zeiten}

So machen Sie Ihr Unternehmen fit für Wachstum und Gewinn

2., überarbeitete und erweiterte Auflage

Springer Gabler 
Hartmut H. Biesel

Dortmund

Deutschland

Die 1. Auflage erschien 2004 unter dem Titel „Turnaround im Vertrieb“.

ISBN 978-3-8349-4615-7

ISBN 978-3-8349-4616-4 (eBook)

DOI 10.1007/978-3-8349-4616-4

Die Deutsche Nationalbibliothek verzeichnet diese Publikation in der Deutschen Nationalbibliografie; detaillierte bibliografische Daten sind im Internet über http://dnb.d-nb.de abrufbar.

Springer Gabler

(C) Springer Fachmedien Wiesbaden 2004, 2014

Das Werk einschließlich aller seiner Teile ist urheberrechtlich geschützt. Jede Verwertung, die nicht ausdrücklich vom Urheberrechtsgesetz zugelassen ist, bedarf der vorherigen Zustimmung des Verlags. Das gilt insbesondere für Vervielfältigungen, Bearbeitungen, Übersetzungen, Mikroverfilmungen und die Einspeicherung und Verarbeitung in elektronischen Systemen.

Die Wiedergabe von Gebrauchsnamen, Handelsnamen, Warenbezeichnungen usw. in diesem Werk berechtigt auch ohne besondere Kennzeichnung nicht zu der Annahme, dass solche Namen im Sinne der Warenzeichen- und Markenschutz-Gesetzgebung als frei zu betrachten wären und daher von jedermann benutzt werden dürften.

\section{Lektorat: Manuela Eckstein}

Gedruckt auf säurefreiem und chlorfrei gebleichtem Papier

Springer Gabler ist eine Marke von Springer DE. Springer DE ist Teil der Fachverlagsgruppe Springer Science+Business Media

www.springer-gabler.de 


\section{Vorwort}

Willkommen in der Welt der Apps! Weltweit nutzen über eine Milliarde Menschen Facebook. Seit 2011 sind die Nutzerzahlen um über $50 \%$ angestiegen. Etwa 350 Millionen Nutzer verwenden mobile Endgeräte, um Facebook zu nutzen, Tendenz stark steigend. $50 \%$ der Nutzer melden sich täglich an. Jeder Benutzer veröffentlicht monatlich durchschnittlich 90 Inhalte, pro Tag sind dies insgesamt über zwei Milliarden neue Einträge.

In Deutschland dominieren zwar immer noch die 18 bis 34-jährigen als Altersgruppe, gerade aber die älteren Menschen weisen die höchsten Wachstumsraten auf. Gemessen an der Benutzerzahl wäre Facebook das drittgrößte Land der Welt. In UK ist bereits die Hälfte des mobilen Internetverkehrs auf Facebook zurückzuführen.

Dabei ist Facebook zwar das größte, aber dennoch weltweit nicht das einzige soziale Netzwerk. Auch andere Netzwerke glänzen mit beeindruckenden Zahlen. So verzeichnet etwa das geschäftlich orientierte Netzwerk LinkedIn jede Sekunde ein neues Mitglied. Alle 60 Sekunden werden mehr als 6000 Bilder auf flickr hochgeladen, knapp 100.000 Tweets über Twitter versandt, 20.000 Postings auf tumblr eingestellt und 600 neue Videos auf YouTube hochgeladen. Angeblich werden weltweit mehr iPhones verkauft als Kinder geboren.

Social Media sind keine Trenderscheinung! Social Business ist eine Sammlung von Methoden und Techniken und verändert als Vernetzungs- und Austauschform die Arbeitsprozesse in den Unternehmen. Das veränderte Kommunikationsverhalten der Menschen wirkt sich auf die Wirtschaft aus. Konsumenten tauschen Meinungen und Erfahrungen in sozialen Netzwerken aus, fragen Bekannte online zu Kaufempfehlungen und negative Erfahrungen verbreiten sich über Nacht weltweit.

Immer mehr Menschen können immer schneller auf dieselben Informationen zugreifen. Es wird entsprechend immer schwieriger, sich durch das Abschirmen von Informationen Machtvorteile zu verschaffen oder je nach Anlass unterschiedlich zu kommunizieren.

Jedes fünfte Paar lernt sich bereits online kennen. Und angeblich sind $20 \%$ der Scheidungen auf Facebook zurückzuführen, weil ein Partner über das Internet eine Jugendbekanntschaft wiedergefunden hat.

In Filmpalästen werden 2,5 Mrd. US\$ für Getränke und Popcorn konsumiert, demgegenüber geben Spieler im Umfeld von Social Media über 6 Mrd. US\$ für virtuelle Güter aus. Starbucks erreicht über die Website monatlich knapp 2 Millionen Besucher, über seine Facebook-Seite 20 Mio. 
Einer weltweiten Studie von Cisco zufolge fragen zwei Drittel aller Studenten bei ihrem neuen Arbeitgeber nach dem Umgang mit Social Media, und mehr als die Hälfte lehnt Angebote von Arbeitgebern ab, bei denen Social Media während der Arbeitszeit verboten sind. Ein Drittel der Absolventen hält die Freiheit in Hinblick auf Social Media, die Wahl von Zugangsgeräten wie Smartphones und flexible Arbeitszeiten und -orte für wichtiger als das Gehalt.

Sebastian Thun, ein deutscher Standford-Professor, hat seine Einstiegsvorlesung zum Thema „Künstliche Intelligenz“ online gestellt und anstelle der geplanten 200 insgesamt 160.000 Teilnehmer erreicht.

Auf Unternehmen hat dies vielfältige Folgen. Social Media werden zu einem wesentlichen Werkzeug in der Positionierung von Unternehmungen und Marken und zu einem selbstverständlichen Bestandteil des Marketing-Mix; darüber hinaus führen sie aufgrund des direkten Rückkanals sowie der Schnelllebigkeit zu neuen Herausforderungen.

Private und geschäftliche Netzwerke verbinden sich zunehmend und werden über Internetplattformen organisiert. Soziale Netzwerke werden die Formen von Kommunikation verändern. Immer mehr Unternehmen starten „Enterprise 2.0“ Initiativen und organisieren die Mitarbeiter, Kunden, Partner und Lieferanten in firmeneigenen, sozialen Netzwerken (ESN = Enterprise Social Network). In kurzer Zeit hat zum Beispiel die BASF über 30.000 Mitarbeitern die Möglichkeit gegeben, sich in themenspezifischen Teams selbst online zu organisieren und auszutauschen. ATOS, mit 80.000 Mitarbeitern und 42 Ländern eines der größten IT-Unternehmen, hat angekündigt, in der firmeninternen Kommunikation künftig gänzlich auf E-Mails zu verzichten und anstelle dessen Soziale Netzwerke zu verwenden. Alcatel Lucent hat binnen einem Jahr 60.000 Mitarbeiter in einem sozialen Netzwerk zusammengeführt, die sich selbst in über 4.000 Gruppen organisieren. Studien von Salesforce.com zufolge führen ESNs zu einem 30 \% geringerem E-Mail-Verkehr, einer um 34\% höheren Mitarbeiterproduktivität und zu 27\% weniger Meetings.

Die Baby-Boomer der Nachkriegsgeneration standen für Aufbau und Sicherheit und waren eher konservativ ausgerichtet. Die X-Generation strebte Karriere und Status an und agierte zielorientiert. Für die Y-Generation der Zukunft wird soziale Vernetzung durch die neuen Medien zum Alltag gehören. Es wird zur Führungsaufgabe, kollektive Intelligenz zu ermöglichen, unentdecktes Wissen freizusetzen und andere Organisationsformen zuzulassen.

Das Pushen und Initiieren von Veränderungsprozessen wird zukünftig mehr denn je zu Ihren Kernaufgaben zählen. Veränderungen erzeugen Spannungen, Stress und manchmal auch Abschiedsschmerzen von liebgewonnenen Ritualen und Spielregeln. Notwendige Veränderungen können Sie nicht verhindern, es sei denn, zu Lasten Ihres Unternehmens und damit auch Ihres Arbeitsplatzes. Über die Höhe des Lustfaktors bei den notwendigen Neuausrichtungen entscheiden Sie selbst erheblich mit.

Selten sind es einzelne Umstände, die eine Neustrukturierung erfordern, sondern der immer schneller werdende Wandel in der Gesellschaft, im Markt, bei Kunden et cetera. Sie verlangen eine Änderung der Denkausrichtung des gesamten Unternehmens und des Vertriebsteams. Wandel wird zur Konstanten. Es ist verführerisch, die sich verändernden 
Grundlagen, etwa die Globalisierung, die Kaufzurückhaltung der ungerechten Kunden, den Wettbewerb oder sonstige höhere Mächte als Ursache für unbefriedigende Unternehmenszahlen verantwortlich zu machen. Es ist leichter, andere für den Veränderungsbedarf anzuprangern, statt zu erkennen, dass der Organismus einer Organisation unabhängig vom Wachstum positiv funktionieren muss.

Es lohnt sich, bei allen Entwicklungen genauer hinzuschauen, um Trends zu erfühlen und zu erkennen, in welche Richtung die Reise geht, damit sich die Vertriebsorganisation flexibel auf die neue Ausrichtung einstellen kann. Einerseits sind einfache Rezepte fehl am Platze, andererseits gibt Ihnen der Markt auch nicht die Zeit, in jahrelanger Kleinarbeit komplexe Strategiemodule mit höchstem Perfektionsanspruch im Unternehmen zu entwickeln und umzusetzen. Eine Balance zu finden zwischen Bewährtem und Weiterentwicklung wird künftig DIE Herausforderung sein.

Gerade der Vertrieb mit seinen internen und externen Kunden unterliegt einem verstärkten Wandel. Die erfolgreiche Gestaltung des Wandels hängt von der Qualität der Vertriebsmannschaften ab. Harte Fakten und ein einheitliches Werteverständnis werden dabei zu wesentlichen Erfolgstreibern, sie beeinflussen die Kooperationsbereitschaft und Gruppendynamik der Unternehmensteams.

Unterschiedliche Reifephasen verlangen unterschiedliche Vorgehensweisen, die Wahl der Strategien und Werkzeuge muss darauf abgestimmt sein. Das Anliegen dieses Buches ist einerseits, das Verständnis für theoretische Überlegungen in pragmatischer und konzentrierter Form zu schärfen, um den Änderungsbedarf zu verdeutlichen. Andererseits möchte es die Sensibilität für die weichen Faktoren steigern und damit die Chancen erhöhen, dass Veränderungsprozesse in kürzerer Zeit unkomplizierter umgesetzt werden. Veränderungsprozesse können nie gegen, sondern nur mit Menschen erfolgreich umgesetzt werden.

Vertriebsorganisationen werden immer wieder aus sachlichen oder persönlichen Gründen ausgebremst. Verfahrensdefizite, mangelnde Methodik oder Kundenorientierung behindern ebenso wie die Angst vor Neuem und Fehlern oder Egozentriertheit.

Das Buch ist für Praktiker aus der Praxis heraus geschrieben und bietet Ihnen viele Umsetzungsideen für ein professionelles Change-Management. Themen wie Mitarbeiterführung und strategisches Vorgehen nehmen in diesem Buch einen breiten Raum ein. Die Qualität der Mitarbeiter und des Wissens werden über den Vertriebserfolg der Zukunft entscheiden.

Für eine kritische Auseinandersetzung mit den Ausführungen bin ich jederzeit dankbar.

Dortmund

Ihr Hartmut Biesel

im Januar 2014 


\section{Inhaltsverzeichnis}

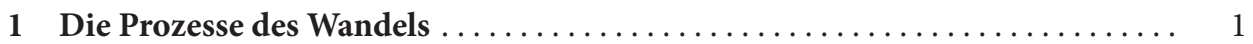

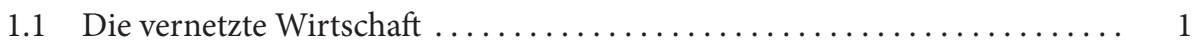

1.2 Ohne Wandel keine Weiterentwicklung $\ldots \ldots \ldots \ldots \ldots \ldots \ldots \ldots \ldots \ldots$

1.3 Der demografische Wandel im Vertrieb ................... 15

1.4 Frühwarnindikatoren rechtzeitig erkennen $\ldots \ldots \ldots \ldots \ldots \ldots \ldots \ldots \ldots$

1.5 Die Zukunftsausrichtung des Vertriebs festlegen ............... 23

1.5.1 Die Einbindung von externen Beratern ............... 30

1.5.2 Ohne Mitarbeiter geht nichts (voran) $\ldots \ldots \ldots \ldots \ldots \ldots \ldots \ldots \ldots \ldots \ldots \ldots$

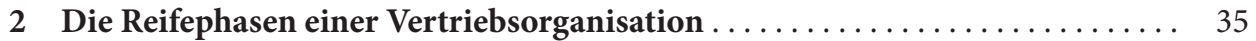

2.1 Die Wachstumsphasen eines Unternehmens . . . . . . . . . . . . 37

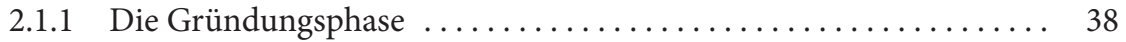

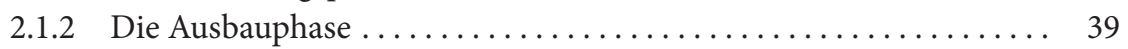

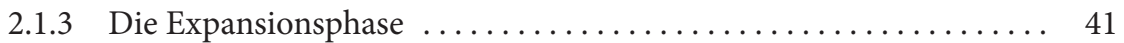

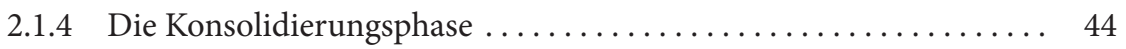

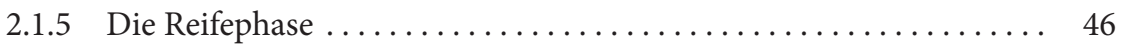

2.2 Die Alterungsphasen eines Unternehmens $\ldots \ldots \ldots \ldots \ldots \ldots \ldots \ldots \ldots . \ldots . \ldots 4$

2.2.1 Die Balancephase $\ldots \ldots \ldots \ldots \ldots \ldots \ldots \ldots \ldots \ldots \ldots \ldots \ldots . \ldots \ldots$

2.2.2 Die Establishmentphase ........................ 49

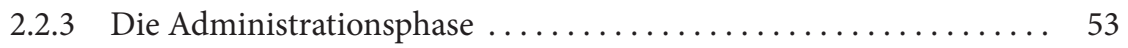

2.3 Das veränderte Verhalten des Vertriebs in den Reifephasen ........... 54

2.3.1 Risikobereitschaft ......................... 55

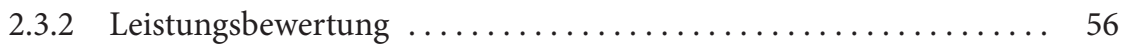

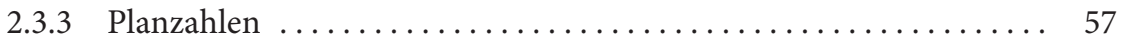

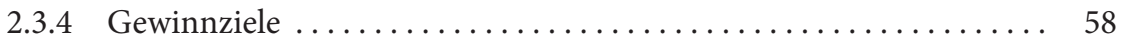

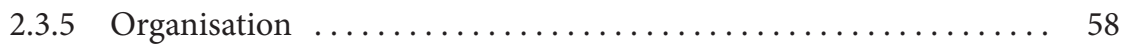

2.4 Die Aufgabe des Vertriebsmanagements im Veränderungsprozess ....... 60

2.4.1 Entscheidungen konsequent durchsetzen ................ 65

2.4.2 Machen Sie Ihr Vertriebsteam zur lernenden Organisation . . . . . . . 69

2.4.3 Die Entwicklung von Machtstrukturen ............... 73 
3 Veränderungsprozesse gestalten und umsetzen $\ldots \ldots \ldots \ldots \ldots \ldots \ldots \ldots .77$

3.1 Voraussetzungen für ein erfolgreiches Veränderungsmanagement . . . . . 77

3.1.1 Überprüfen Sie die Strukturen und Fähigkeiten Ihres Vertriebs . . . 79

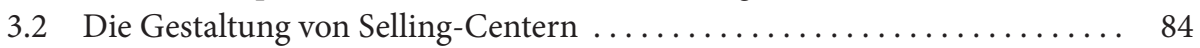

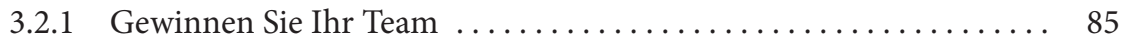

3.2.2 Starten Sie den Veränderungsprozess ................. 91

3.2 .3 Übernehmen Sie die Führung . . . . . . . . . . . . . . . 97

3.3 Erzeugen Sie Vertriebsdynamik . . . . . . . . . . . . . . . 105

3.3.1 Einsatz des Projektmanagements in Veränderungsprozessen .... . 111

3.3.2 Bauen Sie das Wissensmanagement aus ................ 120

3.3.3 Kosten senken durch eine Vertriebsstrukturanalyse .......... 123

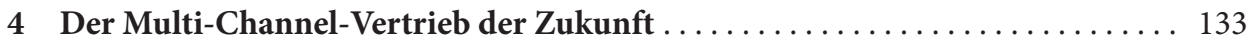

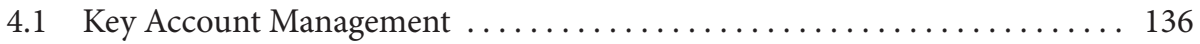

4.2 Der Flächenvertrieb (Außendienst/Vertriebspartner) . . . . . . . . . 138

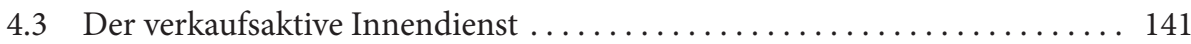

4.4 Organisation einer zentralen Kundenbearbeitung . . . . . . . . . . 145

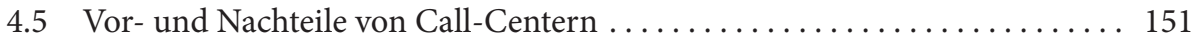

4.6 Die Kundenorientierung im Unternehmen verstärken ............ 153

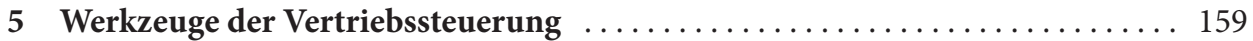

5.1 Konzentration auf Markt und Kunden . . . . . . . . . . . . . . . . . . 159

5.1.1 Strategische Vertriebsziele festlegen - die Vertriebs-Scorecard . . . . 160

5.1.2 Den Kundenwert und Kundenmehrwert definieren ... . . . . . . . . . 169

5.1.3 Markt- und Kundenpotenziale ermitteln ................ 170

5.1.4 Prozesskosten und Kundenlebenswert analysieren ........... 172

5.2 Der Aufbau von Kennzahlensystemen . . . . . . . . . . . . . . . . 174

5.2.1 Neukunden gezielt gewinnen und binden . . . . . . . . . . . 176

5.2.2 Bestimmung der Wettbewerbsposition - Benchmarking . . . . . . . 183

5.3 Aufbau eines Kundenbeziehungsmanagements (CRM) . . . . . . . . . . . 188

5.3.1 Was Sie über Kunden wissen sollten $\ldots \ldots \ldots \ldots \ldots \ldots \ldots \ldots$

5.3.2 Steigern Sie die Kundenzufriedenheit ................. 193

5.3.3 E-CRM gehört die Zukunft . . . . . . . . . . . . . . . . . . . . 197

5.4 Entwicklung zukunftsorientierter Leistungspakete $\ldots \ldots \ldots \ldots \ldots \ldots \ldots$

5.4.1 Beratungs-, Service- und Dienstleistungen ............... 200

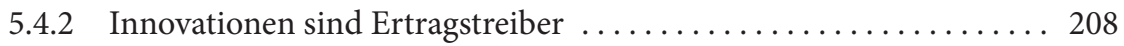

5.4 .3 Preisflexibilität vs. Preisdruck . . . . . . . . . . . . . . . . . . . 215

5.4.4 Gestaltung internationaler Preissysteme .............. 222

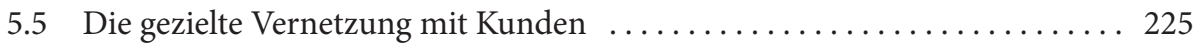

5.5 .1 Supply Chain Management . ..................... 225

5.5.2 Efficient Consumer Response (ECR) ................ 230

5.5 .3 Category Management ....................... 234

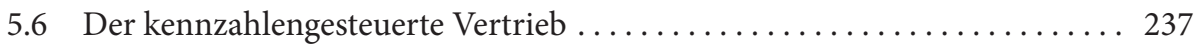


6 Vertriebsmitarbeiter in dynamischen Organisationen führen . . . . . . . . 243

6.1 Mitarbeiter für ehrgeizige Ziele gewinnen $\ldots \ldots \ldots \ldots \ldots \ldots \ldots \ldots \ldots \ldots$

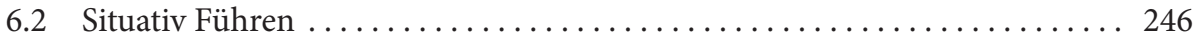

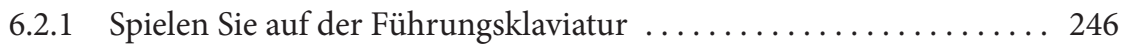

6.2.2 Der gezielte Einsatz von Autorität $\ldots \ldots \ldots \ldots \ldots \ldots \ldots \ldots \ldots \ldots \ldots \ldots$

6.3 Begleiten Sie Ihr Team durch den Veränderungsprozess ............ 256

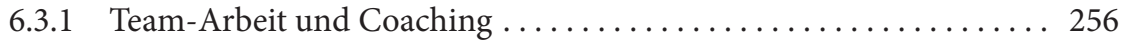

6.3.2 Zielvereinbarungen und Feedback ................. 264

6.4 Der vertrauensbasierte Vertrieb $\ldots \ldots \ldots \ldots \ldots \ldots \ldots \ldots \ldots \ldots \ldots \ldots \ldots \ldots$

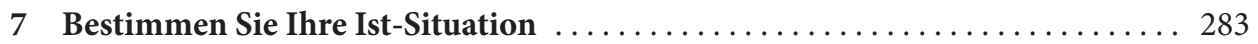

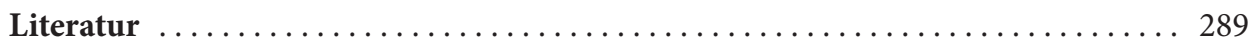




\section{Der Autor}

Hartmut H. Biesel ist geschäftsführender Gesellschafter der APRICOT Unternehmensberatung $\mathrm{GmbH}$ und beschäftigt sich mit den Themen „Neuausrichtung von Vertriebsorganisationen, Entwicklung und Umsetzung von Vertriebsstrategien und Optimierung des Markt- und Kundenmanagements".

Hartmut H. Biesel war 23 Jahre in der Wirtschaft im In- und Ausland tätig, davon 20 Jahre in Management-Positionen. Seit 1997 stellt er seine langjährigen Berufserfahrungen Unternehmen zur Verfügung, die ihre Marketing- und Vertriebsaktivitäten überprüfen und neu ausrichten wollen. Als Berater begleitet er Unternehmen und Führungsverantwortliche bei der Umsetzung eines Turnarounds, er arbeitet als Coach und qualifiziert Mitarbeiter beim Umgang mit neuen Herausforderungen im Kundenmanagement.

Die Herausforderungen in der globalen Wirtschaft erfordern in vielen Unternehmen einen Paradigmenwandel in der Vertriebsausrichtung. Die Weiterentwicklung von Kundenmanagementkonzepten, beispielsweise die Weiterentwicklung von Kundenmanagement-Organisationen, ist deshalb ein wichtiger Schwerpunkt im Beratungs- und Qualifizierungsportfolio von Hartmut $\mathrm{H}$. Biesel ein.

Hartmut H. Biesel ist Mitglied in diversen Organisationen, u. a. Vorstand des „EF-KAM European Foundation for Key Account Management“ und Gründungsmitglied der „Deutschen Gesellschaft für Führungskräfteentwicklung DGFKE e. V.“ Er war Vertriebsexperte des Think Tank „Sales Driven Company“ der Universität St. Gallen und ist erfolgreicher Buchautor, Berater, Trainer, und Coach. 